\section{ACTIVELY STRENGTHENING AND RE-HARDENING ENAMEL}

This year the GSK Consumer Healthcare team will be visiting the British Dental Conference and Exhibition to showcase Sensodyne and Pronamel.

New Sensodyne Repair \& Protect - now with stronger repair - offers sensitivity sufferers the benefit of both advanced NovaMin technology and sodium fluoride in a single formulation. The immediate availability of sodium fluoride when brushing allows for greater fluoride uptake into the hydroxyapatite-like layer formed by NovaMin over exposed dentine.

Pronamel toothpaste has an optimised fluoride formulation to help minerals penetrate deep into the tooth surface, actively strengthening and re-hardening acid-weakened enamel. Pronamel toothpaste delivers more fluoride deeper into enamel than other fluoride toothpaste helping to protect patients enamel from the effects of erosive tooth wear.

Visit stand B03 to try Sensodyne Repair \& Protect and Pronamel toothpaste for yourself in GSK's tasting station. The stand will also feature an interactive lecture revealing the truth about patients with dentine hypersensitivity and erosive tooth wear.

GSK are pleased to be sponsoring two presentations at the conference:

'Burden of Oral Diseases in Ageing Populations and links with General Health' - Georgios Tsakos, Thursday 26 May, 14:30 - 15:30 pm

'How to Manage and Prevent Patient Tooth Wear Caused by Dietary Acids' - Rupert Austin, Friday 27 May, 11:45 - 12:45 pm

For further information on Sensodyne and Pronamel visit stand B03 or www.gsk-dentalprofessionals.co.uk.

\title{
SUPPORT YOUR DENTURE PATIENTS
}

When patients hear the news that they need dentures, everything else you tell them is often unheard. At least 32\% of patients don't know what questions to ask their dentist when getting their new denture fitted (based on a survey of 1,748 denture wearers in four countries).

To help support your patients on their denture journey, the Poligrip Partner Programme has been created in conjunction with dental professionals.

Ask your GSK representative today for further information on the Poligrip range and Partner Programme.

The Poligrip range includes:

- Poligrip Overnight Whitening Daily Cleanser

- Poligrip 3 Minute Daily Cleanser

- Poligrip Ultra Fixative Cream

- Poligrip Flavour Free Fixative Cream.

Recommend the Poligrip range to help your patients with dentures eat, speak and smile with confidence.

For additional support GSK have developed a distance learner module on the topic of 'Caring for Patients with Dentures'. The module has been designed to provide you with an insight into the patient experience of dentures as well as supporting you when providing practical advice.

For further information on the Poligrip range and to complete the distance learner module visit www.gskdentalprofessionals.co.uk. Completion of the module can contribute up to 1.5 hours towards your verifiable CPD.
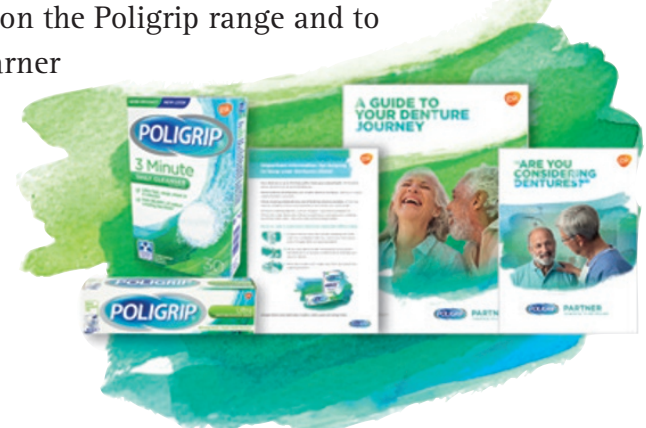

\section{MIND THE SMALLER GAP}

TePe has launched a new TePe EasyPick size. Colour coded Orange, the smaller size is designed to fit extra small and small gaps and offers an alternative to the EasyPick Turquoise, designed for slightly larger gaps. Like its partner, the TePe EasyPick Orange is easy to use, made from silicone and ergonomically designed to be easily gripped.

The design of the EasyPick Orange allows the user to access smaller gaps between the teeth, whilst its tapered head helps remove trapped food and any built-up plaque from between the teeth - helping to improve overall dental health. EasyPick is now available to practices.

TePe EasyPick is comfortable to use, gentle on the gums and has a non-slip grip. It has been designed for on-the-go use to provide a cost-effective solution to remove trapped food and also to help patients reach the hidden $40 \%$ of the tooth that regular tooth brushing misses.

TePe EasyPick Orange and Turquoise come in a re-sealable pack of 36 and each pack includes a handy travel case to encourage frequent, on-the-go use. Ideal for encouraging patient compliance and healthy habit formation.

www.tepe-easypick.com

\section{OPTIMAL SALIVA CONTROL DURING PROCEDURES}

Super absorbents have reached a new level with the introduction of the new and unique Drydent Sublingual for optimal saliva control.

Drydent Sublingual is a unique new product which collects and controls the saliva produced by the sublingual glands as well as the submandibular glands.

It is placed under the tongue for the optimal collection of unwanted moisture under the sublingual area. Its super absorbent qualities provide a super dry oral environment during a variety of procedures.

Patient comfort has been improved with its smooth soft material design that reduces uncomfortable swallowing reflexes. Saliva ejectors can be placed over the DryDent Sublingual, minimising uncomfortable suctions of soft tissue. DryDent Sublingual's super absorbent capacity keeps moisture control even when it is fully saturated.

http://www.directadental.com/exego.aspx?p_id=1861 